\title{
THE QUIESCENT X-RAY PROPERTIES OF THE ACCRETING MILLISECOND X-RAY PULSAR AND ECLIPSING BINARY SWIFT J1749.4-2807
}

\author{
N. DegenaAR ${ }^{1,3}$, A. Patruno ${ }^{2}$, ANd R. Wijnands ${ }^{2}$ \\ ${ }^{1}$ Department of Astronomy, University of Michigan, 500 Church Street, Ann Arbor, MI 48109, USA; degenaar@umich.edu \\ 2 Astronomical Institute "Anton Pannekoek," University of Amsterdam, Postbus 94249, 1090 GE Amsterdam, The Netherlands \\ Received 2012 April 27; accepted 2012 July 13; published 2012 August 23
}

\begin{abstract}
Swift J1749.4-2807 is a transient neutron star low-mass X-ray binary that contains an accreting millisecond X-ray pulsar spinning at $518 \mathrm{~Hz}$. It is the first of its kind that displays X-ray eclipses, which holds significant promise to precisely constrain the mass of the neutron star. We report on a $\simeq 105 \mathrm{ks}$ long XMM-Newton observation performed when Swift J1749.4-2807 was in quiescence. We detect the source at a $0.5-10 \mathrm{keV}$ luminosity of $\simeq 1 \times 10^{33}(D / 6.7 \mathrm{kpc})^{2} \mathrm{erg} \mathrm{s}^{-1}$. The X-ray light curve displays three eclipses that are consistent in orbital phase and duration with the ephemeris derived during outburst. Unlike most quiescent neutron stars, the X-ray spectrum can be adequately described with a simple power law, while a pure-hydrogen atmosphere model does not fit the data. We place an upper limit on the $0.01-100 \mathrm{keV}$ thermal luminosity of the cooling neutron star of $\lesssim 2 \times 10^{33} \mathrm{erg} \mathrm{s}^{-1}$ and constrain its temperature to be $\lesssim 0.1 \mathrm{keV}$ (for an observer at infinity). Timing analysis does not reveal evidence for X-ray pulsations near the known spin frequency of the neutron star or its first overtone with a fractional rms of $\lesssim 34 \%$ and $\lesssim 28 \%$, respectively. We discuss the implications of our findings for dynamical mass measurements, the thermal state of the neutron star, and the origin of the quiescent X-ray emission.
\end{abstract}

Key words: binaries: eclipsing - pulsars: general - pulsars: individual (Swift J1749.4-2807) - stars: neutron $\mathrm{X}$-rays: binaries

\section{INTRODUCTION}

Neutron stars in low-mass X-ray binaries (LMXBs) accrete matter from a sub-solar $\left(\lesssim 1 M_{\odot}\right)$ companion star that overflows its Roche-lobe. Such binaries are generally thought to be very old $\left(\sim 10^{9} \mathrm{yr}\right)$ and their neutron stars may have gained substantial mass during their evolution via accretion. Studying LMXBs therefore holds great promise to probe the high end of the mass distribution of neutron stars, which provides strong constraints on the equation of state of ultra-dense matter (Lattimer \& Prakash 2001).

Many LMXBs are transient and spend the majority of their time in a dim, quiescent state at a $0.5-10 \mathrm{keV}$ luminosity of $L_{\mathrm{X}} \sim 10^{31-33} \mathrm{erg} \mathrm{s}^{-1}$ (e.g., Jonker et al. 2004). They exhibit occasional X-ray outbursts during which their intensity rises to $L_{\mathrm{X}} \sim 10^{36-38} \mathrm{erg} \mathrm{s}^{-1}$, and that typically last a few weeks or months (e.g., Chen et al. 1997). The outbursts result from a sudden strong increase in the mass-accretion rate onto the neutron star, whereas little or no accretion takes place in quiescence.

Accreting millisecond X-ray pulsars (AMXPs) form a small (counting 14 members to date) subclass of transient LMXBs that display coherent X-ray pulsations with a frequency of 182-599 Hz (Wijnands 2006; Patruno 2010). Their magnetic field of $B \sim 10^{8-9} \mathrm{G}$ is strong enough to concentrate the accretion flow onto the magnetic poles of the neutron star, which creates local hotspots and gives rise to the distinctive $\mathrm{X}$-ray pulsations.

\subsection{Swift J1749.4-2807}

Swift J1749.4-2807 was discovered with Swift on 2006 June 2 when it exhibited a thermonuclear X-ray burst (Wijnands et al. 2009 and references therein). The observed burst peak put the

\footnotetext{
3 Hubble fellow.
}

source at a distance of $D \lesssim 6.7 \mathrm{kpc}$. Its $0.5-10 \mathrm{keV}$ luminosity rapidly declined from $L_{\mathrm{X}} \approx 10^{36}$ to $\sim 10^{33}(D / 6.7 \mathrm{kpc})^{2} \mathrm{erg} \mathrm{s}^{-1}$ within one day after its discovery (Beardmore et al. 2006; Wijnands et al. 2009). The source was serendipitously detected in quiescence at $L_{\mathrm{X}} \sim 10^{33}(D / 6.7 \mathrm{kpc})^{2} \mathrm{erg} \mathrm{s}^{-1}$ during three archival XMM-Newton observations performed in 2000 and 2006 (Halpern 2006). However, it was located far off-axis, inhibiting an accurate determination of the X-ray flux and spectrum (Wijnands et al. 2009).

On 2010 April 10, renewed activity was detected from Swift J1749.4-2807 with INTEGRAL (Pavan et al. 2010). The outburst lasted for $\simeq 11$ days and had an average $0.5-10 \mathrm{keV}$ luminosity of $L_{\mathrm{X}} \sim 10^{36}\left(D / 6.7 \mathrm{kpc}\right.$ ) $\mathrm{erg} \mathrm{s}^{-1}$ (Altamirano et al. 2011; Ferrigno et al. 2011). The discovery of $518 \mathrm{~Hz}$ coherent X-ray pulsations with RXTE identified the source as a new AMXP (Altamirano et al. 2010). It is the first of its class to display $\mathrm{X}$-ray eclipses, which occur when the companion star moves into our line of sight and obscures the central X-ray source. The eclipses recur at the $8.82 \mathrm{hr}$ orbital period of the binary (Markwardt et al. 2010; Markwardt \& Strohmayer 2010).

The mass donor in Swift J1749.4-2807 is a main-sequence star of spectral type K-G (Markwardt \& Strohmayer 2010; D'Avanzo et al. 2011). This holds good prospects for studying the radial velocity curve of the companion in quiescence. The detection of X-ray eclipses tightly constrains the binary inclination to $i \simeq 74^{\circ}-78^{\circ}$, which provides a key ingredient for such studies (Markwardt \& Strohmayer 2010).

Investigation of the quiescent X-ray properties is an important aspect of the challenge to accurately constrain the neutron star mass. The quiescent luminosity provides the diagnostic to determine if the donor star suffers from X-ray irradiation, which would affect its radial velocity curve (e.g., Bassa et al. 2009). Furthermore, the X-ray spectrum of quiescent LMXBs can be used to constrain the neutron star temperature, which offers an alternative way to probe the equation of state (e.g., Wijnands et al. 2001; Yakovlev et al. 2003; Page et al. 2004; Heinke et al. 


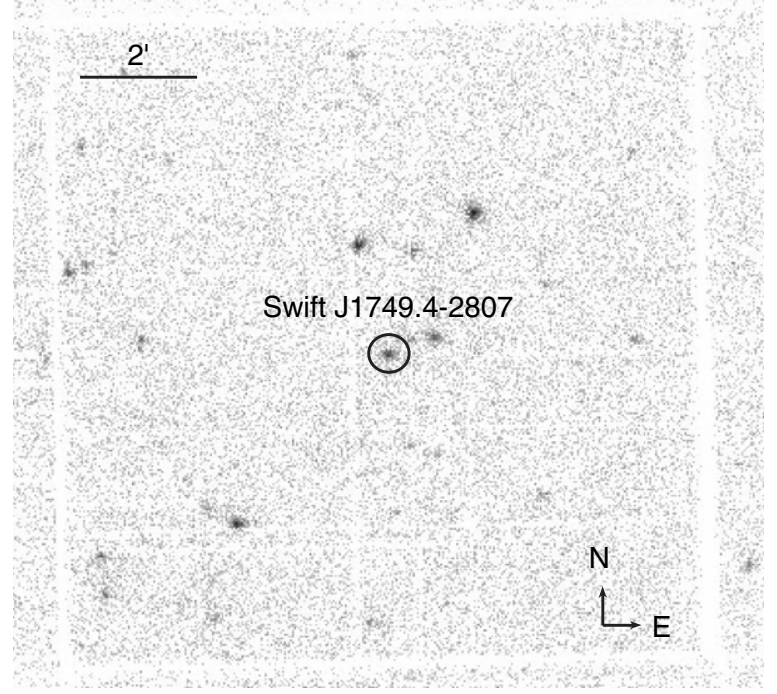

Figure 1. Combined MOS1/MOS2 X-ray image $(0.3-12 \mathrm{keV})$ of the field around Swift J1749.4-2807.

2009). In this paper, we report on a long pointed XMM-Newton observation of Swift J1749.4-2807 in quiescence.

\section{OBSERVATIONS, DATA REDUCTION, AND RESULTS}

Swift J1749.4-2807 was observed with XMM-Newton for $\simeq 105 \mathrm{ks}$ from UT 2011 March 19 16:25 till March 20 21:30 (ObsID 0655670101). We used the data obtained with the European Photon Imaging Camera (EPIC). This instrument consists of two MOS detectors (each made up of an array of 7 CCDs; Turner et al. 2001) and one PN camera (an array of 12 CCDs; Strüder et al. 2001).

Both MOS cameras were operated in the full window imaging mode. The PN was set in timing mode, in which all the two-dimensional spatial information is collapsed into a single dimension, providing a time resolution of $30 \mu \mathrm{s}$. Data reduction and analysis was carried out using the Science Analysis Software (sAs; ver. 11.0.0).

Part of the data was affected by background flares, particularly near the end of the observation. We excluded such episodes by selecting only data with high-energy count rates of $<0.35$ counts s $^{-1}$ for the MOS and $<0.20$ counts $\mathrm{s}^{-1}$ for the PN. This resulted in a good exposure time of $\simeq 80$ and $\simeq 74 \mathrm{ks}$ for the two MOS and the PN, respectively.

\subsection{X-Ray Image and Light Curve}

Figure 1 displays the combined MOS1/MOS2 image zoomed in on the central CCD. Swift J1749.4-2807 is clearly detected and among the brightest sources in the field. Using the tool EDETECT_CHAIN, we obtain a position of right ascension (R.A.) $17^{\mathrm{h}} 49^{\mathrm{m}} 31^{\mathrm{s}} .58$ and declination (decl.) $-28^{\circ} 08^{\prime} 04^{\prime \prime} .5$ (J2000.0). Combining the 0.7 statistical uncertainty of the detection routine with the estimated 1".5 MOS systematic error (Watson et al. 2009), yields a positional uncertainty of $1^{\prime \prime} .7$ (90\% confidence). The XMM-Newton coordinates are offset by $\simeq 2$ ".9-3."3 from the 1".6-1".9 Swift localization (90\% confidence; Yang et al. 2010; D’Avanzo et al. 2011), but consistent within the errors.

We used a circular region with a radius of $12^{\prime \prime}$ to extract source events, and a 36" circular region placed on a sourcefree part of the CCD for the background. Swift J1749.4-2807 is detected in the MOS at a factor $\simeq 3$ above the background

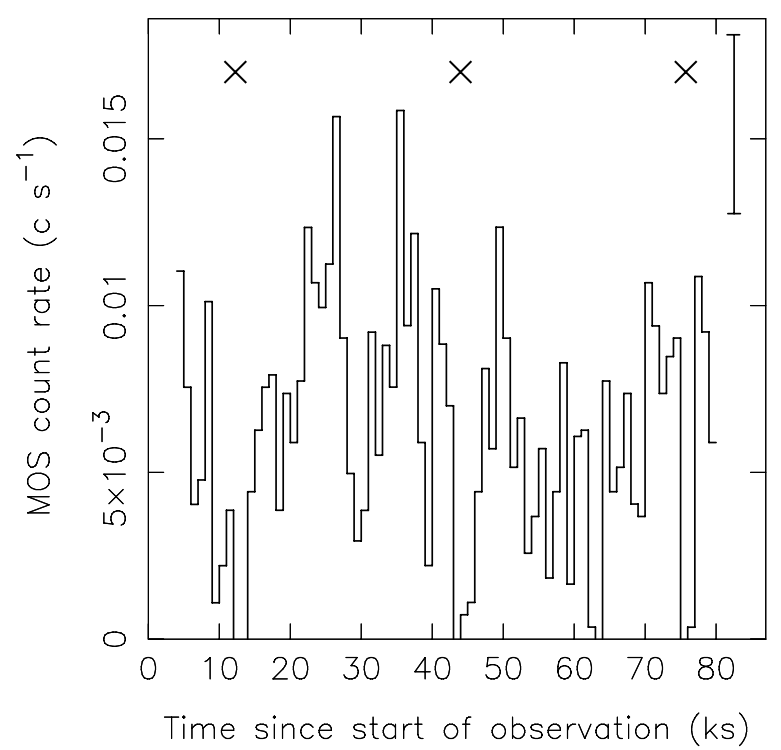

Figure 2. Summed MOS1/MOS2 X-ray light curve at $1000 \mathrm{~s}$ resolution (2-8 keV). The expected times of eclipses are marked by crosses. A representative error bar ( $90 \%$ confidence) is shown in the upper right corner.

$\left(\simeq 1.4 \times 10^{-3}\right.$ counts $\left.\mathrm{s}^{-1}\right)$ with an average net source count rate of $(3.3 \pm 0.3) \times 10^{-3}$ counts $\mathrm{s}^{-1}(0.3-12 \mathrm{keV})$.

We created background-corrected light curves for the MOS data using the tasks EVSELECT and LCCORR. To optimize the signal-to-noise ratio $(\mathrm{S} / \mathrm{N})$, we only selected events between 2 and $8 \mathrm{keV}$, since the energy spectrum of Swift J1749.4-2807 peaks in this range (Section 2.2). Figure 2 displays the summed MOS1/MOS2 light curve, which covers MJD 55639.684-55640.896. There are three strong drops in intensity that last $\simeq 2 \mathrm{ks}$ and occur at times when the source was expected to be eclipsed based on its orbital ephemeris (marked by crosses in Figure 2; Markwardt \& Strohmayer 2010). The observed times of the eclipses are consistent with the predicted ones within the resolution of the light curve (500 s). This firmly establishes that we have detected the quiescent counterpart of Swift J1749.4-2807. During the eclipses, the source count rate drops $\simeq 2 \sigma$ below the mean and becomes consistent with zero, so no residual source flux is detected.

Apart from the eclipses, there are other instances at which the source intensity drops $\simeq 1 \sigma-2 \sigma$ below the mean count rate (Figure 2). There are, however, also several data points during which the count rate is $>1.5 \sigma$ above the mean. This indicates that the additional intensity dips likely result from fluctuations in the data.

\subsection{Spectral Analysis}

We extracted X-ray spectra from the MOS data using the task ESPECGET, using the same source and background regions as employed for the extraction of count rates and light curves (Section 2.1). This tool also generates the appropriate ancillary response files and redistribution matrix files. We combined the two MOS spectra and the weighted response files. ${ }^{4}$

The spectral data were grouped to contain a minimum of 15 photons per bin and fit in the $0.5-10 \mathrm{keV}$ energy range using XSPEC (v. 12.7.0; Arnaud 1996). To calculate the correct non-eclipsed X-ray fluxes, we reduced the exposure time of the

\footnotetext{
4 See xmm.esac.esa.int/sas/current/documentation/threads/ epic_merging.shtml.
} 
Table 1

Spectral Results

\begin{tabular}{lccc}
\hline \hline Parameter (unit) & BBODYRAD & POWERLAW & POWERLAW+NSATMOS \\
\hline$N_{\mathrm{H}}\left(\times 10^{22} \mathrm{~cm}^{-2}\right)$ & $3.6 \pm 1.8$ & $6.7 \pm 3.2$ & $7.3 \pm 4.0 \mathrm{fix}$ \\
$\Gamma$ & $\ldots$ & $1.6 \pm 0.8$ & $1.6 \pm 0.8$ \\
$k T(\mathrm{keV})$ & $1.6 \pm 0.4$ & $\ldots$ & $<0.1$ \\
$R(D / 6.7 \mathrm{kpc} \mathrm{km})$ & $0.03_{-0.03}^{+1.85}$ & $\ldots$ & $10 \mathrm{fix}$ \\
$\chi_{v}^{2} /$ dof & $1.0 / 24$ & $1.0 / 24$ & $1.0 / 23$ \\
$F_{\mathrm{X}, \text { abs }}\left(\times 10^{-13} \mathrm{erg} \mathrm{cm}^{-2} \mathrm{~s}^{-1}\right)$ & $1.0 \pm 0.2$ & $1.1 \pm 0.2$ & $1.1 \pm 0.1$ \\
$F_{\mathrm{X}, \text { unabs }}\left(\times 10^{-13} \mathrm{erg} \mathrm{cm}^{-2} \mathrm{~s}^{-1}\right)$ & $1.3 \pm 0.1$ & $2.5 \pm 0.4$ & $3.2 \pm 0.4$ \\
$L_{\mathrm{X}}\left(\times 10^{33}\left[D / 6.7 \mathrm{kpc}^{2} \mathrm{erg} \mathrm{s}^{-1}\right)\right.$ & $0.7 \pm 0.1$ & $1.3 \pm 0.3$ & $1.7 \pm 0.2$ \\
$L_{\text {th, bol }}\left(\times 10^{33}\left[D / 6.7 \mathrm{kpc}^{2} \mathrm{erg} \mathrm{s}^{-1}\right)\right.$ & $0.9 \pm 0.1$ & $\ldots$ & $<2$ \\
Thermal fraction & $\ldots$ & $\ldots$ & $<55 \%$ \\
\hline
\end{tabular}

Notes. Quoted errors represent $90 \%$ confidence levels. The temperature is for an observer at infinity. $F_{\mathrm{X} \text {,abs }}$ and $F_{\mathrm{X} \text {,unabs }}$ represent the $0.5-10 \mathrm{keV}$ absorbed and unabsorbed flux, respectively. $L_{\mathrm{q}}$ denotes the $0.5-10 \mathrm{keV}$ luminosity and $L_{\text {th, bol }}$ the $0.01-100 \mathrm{keV}$ thermal luminosity. The bottom row gives the fractional contribution of the thermal component to the total unabsorbed $0.5-10 \mathrm{keV}$ model flux.

averaged spectrum by $6500 \mathrm{~s}$ to take into account the presence of three eclipses (Markwardt \& Strohmayer 2010). In all fits, we include interstellar absorption along the line of sight by using the PHABS model with the default XSPEC-abundances and cross-sections (Anders \& Grevesse 1989; Church et al. 1997). The results of our spectral analysis are summarized in Table 1. Quoted errors refer to $90 \%$ confidence levels.

We first attempted to fit the spectral data with a purehydrogen $(\mathrm{H})$ neutron star atmosphere model. We chose the model NSATMOS, which consists of five parameters: the temperature, mass, and radius of the neutron star, the source distance, and a normalization parameter that reflects the fraction of the neutron star surface that is emitting (Heinke et al. 2006). We explored fits by fixing the model parameters to different values or leaving them free to float. All trials resulted in reduced chisquared values of $\chi_{v}^{2}>2.4$ for 21-26 degrees of freedom (dof). We thus conclude that an $\mathrm{H}$-atmosphere model does not provide an adequate description of the spectral data.

A simple blackbody model (BBODYRAD) does provide an acceptable fit, yielding a hydrogen column density of $N_{\mathrm{H}}=$ $(3.6 \pm 1.8) \times 10^{22} \mathrm{~cm}^{-2}$, a temperature of $k T=1.6 \pm 0.4 \mathrm{keV}$, and an emitting radius of $R=0.03_{-0.03}^{+1.85} \mathrm{~km}$ for an assumed distance of $D=6.7 \mathrm{kpc}$ (resulting in $\chi_{v}^{2}=1.0$ for 24 dof; Table 1). The obtained hydrogen column density is similar to the outburst value $\left(N_{\mathrm{H}}=3.0 \times 10^{22} \mathrm{~cm}^{-2}\right.$; Yang et al. 2010; Ferrigno et al. 2011), but the best-fit temperature is considerably higher than that typically found for blackbody fits of quiescent, thermally emitting neutron stars $(k T \simeq 0.1-0.2 \mathrm{keV}$; e.g., Rutledge et al. 1999; Wijnands et al. 2003). This may indicate that the emission is rather non-thermal.

The data can also be fit with a single power-law model (POWERLAW), resulting in $N_{\mathrm{H}}=(6.7 \pm 3.2) \times 10^{22} \mathrm{~cm}^{-2}$ and a photon index of $\Gamma=1.6 \pm 0.8\left(\chi_{v}^{2}=1.0\right.$ for 24 dof; Table 1$)$. This spectral shape is similar to that found for other AMXPs in quiescence (see Section 3.3). Although the obtained hydrogen column density is a factor $>2$ higher than found during outburst, the values are consistent within the errors. Figure 3 displays the combined MOS spectrum of Swift J1749.4-2807 along with the power-law fit.

To obtain upper limits on any thermal emission due to the cooling neutron star (see Section 3.2), we added an NSATMOS component to the best-fit power-law model. We fixed the neutron star mass and radius to $M=1.4 M_{\odot}$ and $R=10 \mathrm{~km}$, adopt $D=6.7 \mathrm{kpc}$, and set the normalization to 1 (Heinke et al. 2006),

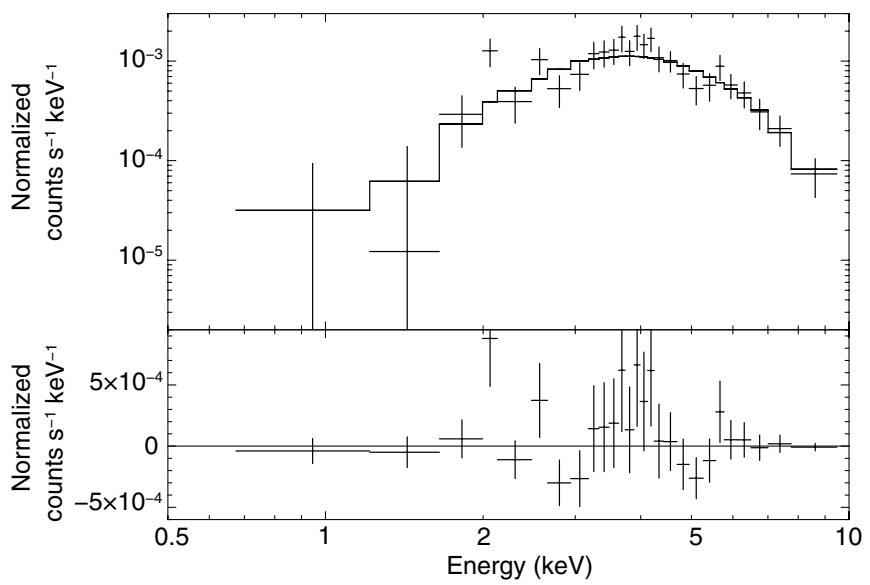

Figure 3. Combined MOS1/MOS2 X-ray spectrum (top) and fit residuals (bottom). The solid curve represents the best fit to a single power-law model.

leaving the temperature as the only free-fit parameter. This resulted in an upper limit on the neutron star temperature (as seen by a distant observer) of $k T \lesssim 0.1 \mathrm{keV}$ and a thermal bolometric luminosity (obtained by extrapolating the thermal model fit to the 0.01-100 keV range) of $L_{\text {th,bol }} \lesssim 2 \times 10^{33} \mathrm{erg} \mathrm{s}^{-1}$. For this fit, the thermal contribution to the total unabsorbed $0.5-10 \mathrm{keV}$ model flux is $\lesssim 55 \%$ (90\% confidence; Table 1 ).

There are hints of possible absorption features (near 3 and $5 \mathrm{keV}$; Figure 3), which are also seen in the two individual MOS spectra. To our knowledge, there are no known instrumental features in this energy range. To estimate the significance, we add a Gaussian absorption line (GABS) to the broadband spectral model. This yields a central energy of $E_{\mathrm{c}}=5.1 \pm 0.3 \mathrm{keV}$ and a width of $0.3 \pm 0.3 \mathrm{keV}$. This implies that it is significant only at $\mathrm{a} \simeq 2 \sigma$ level (defined as the width of the line divided by its $1 \sigma$ statistical error). It has been hypothesized that gravitationally redshifted metal absorption lines may occur in the X-ray spectra of quiescent neutron stars if accretion continues at a low rate (Brown et al. 1998). However, such features have not been established in neutron star LMXBs with well-studied quiescent spectra.

\subsection{Timing Analysis}

We determined the location of Swift J1749.4-2807 in the PN timing data using the tool ESKY2DET and accordingly extracted 
events using a rectangular region of $5 \times 199$ pixels covering the columns RAWX $=37-41$. A box of $5 \times 199$ pixels placed on RAWX $=12-16$ served as our background reference. We used the 2-8 keV energy range to optimize the SNR ratio (Section 2.2).

The data were folded in a single profile of eight bins by using the pulsar ephemeris reported in Altamirano et al. (2011). The ephemeris has a precision $\left(2 \times 10^{-8}\right.$ days $)$ that is sufficient to propagate the orbital and spin solution to the XMM-Newton observation epoch $(\simeq 340$ days after the ephemeris reference time). After creating the pulse profile, we removed $\simeq 3100$ background photons and were left with $\simeq 550$ photons coming from the AMXP. We then fitted a sinusoid plus a constant and calculated the fractional rms amplitude of the pulsation. We repeated the folding procedure at twice the pulsar frequency to inspect the presence of a first overtone.

We did not detect pulsations with an $\mathrm{S} / \mathrm{N}>3$ (defined as the ratio between the pulse amplitude and its $1 \sigma$ statistical error). The $95 \%$ confidence level upper limits are $34 \%$ and $28 \%$ rms for the fundamental and the first overtone, respectively. In broader energy bands, the data are dominated by background noise, hence no constraining upper limits could be obtained.

\section{DISCUSSION}

We report on a long pointed XMM-Newton observation of Swift J1749.4-2807 in quiescence, performed $\simeq 11$ months after the end of its 2010 outburst. The source is clearly detected during our observation and we infer a $0.5-10 \mathrm{keV}$ quiescent luminosity of $L_{\mathrm{X}} \simeq 1 \times 10^{33}(\mathrm{D} / 6.7 \mathrm{kpc})^{2} \mathrm{erg} \mathrm{s}^{-1}$. This is similar to the source intensity estimated from archival XMM-Newton data, which suggests that no strong quiescent variability occurred between 2000, 2006, and 2011 (cf. Wijnands et al. 2009). The $\mathrm{X}$-ray light curve shows three eclipses that are consistent in orbital phase and duration with the ephemeris derived during outburst (Markwardt \& Strohmayer 2010).

The quiescent X-ray spectrum of Swift J1749.4-2807 is highly absorbed $\left(N_{\mathrm{H}} \gtrsim 3 \times 10^{22} \mathrm{~cm}^{-2}\right)$ and peaks around $\simeq 4 \mathrm{keV}$. The spectral data are best fit by either a single power law with an index of $\Gamma \simeq 1.6$ or a blackbody with a temperature of $k T \simeq 1.6 \mathrm{keV}$ and an emitting radius of $R \lesssim 2 \mathrm{~km}$. A pure-H atmosphere model, which usually fits the X-ray spectra of quiescent neutron star LMXBs well, does not provide an adequate description of the data. By adding an NSATMOS component to the best-fit power-law model, we obtain an upper limit on the neutron star temperature of $k T \lesssim 0.1 \mathrm{keV}$ and on its thermal bolometric luminosity of $L_{\text {th, bol }} \lesssim 2 \times 10^{33} \mathrm{erg} \mathrm{s}^{-1}$.

Timing analysis did not reveal evidence for X-ray pulsations near the known spin frequency of the neutron star or its first overtone with upper limits on their rms amplitudes of $34 \%$ and $28 \%$, respectively. During outburst, the pulsations were detected with rms amplitudes of $\simeq 6 \%-29 \%$ (fundamental) and $\simeq 6 \%-23 \%$ (first overtone; Altamirano et al. 2011). Therefore, our upper limits do not exclude that X-ray pulsations occur in quiescence.

\subsection{Implications for Dynamical Mass Measurements}

We can asses whether X-ray irradiation may affect the radial velocity curve of the companion star in quiescence. For a binary separation of $\simeq 2 \times 10^{11} \mathrm{~cm}$ (Markwardt \& Strohmayer 2010), and an isotropic X-ray luminosity of $L_{\mathrm{X}} \simeq 10^{33} \mathrm{erg} \mathrm{s}^{-1}$ at normal incidence, we estimate an irradiation temperature at the companion star of $\simeq 2400 \mathrm{~K}$. We consider this an upper limit, since thermal reprocessing of X-rays is likely $<100 \%$ efficient and the quiescent accretion disk may partly shield the companion from incident X-rays.

Isolated main-sequence stars of spectral type $\mathrm{K}-\mathrm{G}$ have an effective temperature in the range of $\simeq 3000-6000 \mathrm{~K}$ (Tokunaga 2000). Donor stars that have experienced mass loss and/or are rapidly rotating can, however, have a substantially lower temperature. The estimated irradiation temperature is likely too small to play an important role when compared to isolated $\mathrm{K}-\mathrm{G}$ stars, but binary evolution calculations are required to asses this question robustly for the companion of Swift J1749.4-2807.

\subsection{Thermal Emission Due to Deep Crustal Heating}

According to the deep crustal heating model, a neutron star is heated due to a chain of nuclear reactions that take place in the crust during outburst episodes. When accretion switches off in quiescence, the hot neutron star will thermally radiate its heat (Brown et al. 1998). Many neutron star LMXBs display soft $(\lesssim 2 \mathrm{keV})$ quiescent $\mathrm{X}$-ray emission that can readily be described by a neutron star atmosphere model. The deep crustal heating mechanism is generally accepted as the most promising interpretation of this soft thermal emission.

The quiescent X-ray spectrum of Swift J1749.4-2807 cannot be described by a (pure $\mathrm{H}$ ) neutron star atmosphere model. This suggests that we do not detect thermal emission from the hot, cooling neutron star. Based on the outburst history of Swift J1749.4-2807, we can estimate the thermal quiescent emission that is expected to arise from deep crustal heating and compare this with our obtained upper limit.

The mechanism predicts a quiescent thermal luminosity that depends on the accretion history of the binary as $L_{\text {th,bol }}=$ $\langle\dot{M}\rangle Q_{\text {nuc }} / m_{\mathrm{u}} \simeq 1.9 \times 10^{18}\langle\dot{M}\rangle \mathrm{erg} \mathrm{s}^{-1}$ (Brown et al. 1998). Here, $Q_{\text {nuc }} \simeq 2 \mathrm{MeV}$ is the energy released in the crust per accreted nucleon (Gupta et al. 2007; Haensel \& Zdunik 2008, but see Degenaar et al. 2011), $m_{\mathrm{u}}$ is the atomic mass unit, and $\langle\dot{M}\rangle$ is the mass accretion rate onto the neutron star averaged over $\simeq 10^{4} \mathrm{yr}$. The latter can be calculated by multiplying the average accretion-rate during outburst, $\left\langle\dot{M}_{\mathrm{ob}}\right\rangle$, with the duty cycle of the binary (i.e., the ratio of the average outburst duration and the recurrence time).

The outbursts of Swift J1749.4-2807 appear to be short, although the exact duration is not well constrained (Wijnands et al. 2009; Ferrigno et al. 2011, see also Section 1.1). The average outburst intensity of $L_{\mathrm{X}} \simeq 10^{36} \mathrm{erg} \mathrm{s}^{-1}$ suggests an accretion rate of $\left\langle\dot{M}_{\mathrm{ob}}\right\rangle=R L / G M \simeq 8 \times 10^{-11} M_{\odot} \mathrm{yr}^{-1}$ (for $R=10 \mathrm{~km}$ and $M=1.4 M_{\odot}$ ). An outburst length of $t_{\mathrm{ob}}=2$ weeks and a recurrence time of $t_{\mathrm{rec}}=4 \mathrm{yr}$ would imply a duty cycle of $1 \%$, and a time-averaged mass-accretion rate of $\langle\dot{M}\rangle=\left\langle\dot{M}_{\mathrm{ob}}\right\rangle \times t_{\mathrm{ob}} / t_{\mathrm{rec}} \simeq 8 \times 10^{-13} M_{\odot} \mathrm{yr}^{-1}\left(\simeq 5 \times 10^{13} \mathrm{~g} \mathrm{~s}^{-1}\right)$. If this is representative for the long-term accretion history of the binary, then the expected quiescent thermal luminosity is $L_{\text {th,bol }} \simeq 10^{32} \mathrm{erg} \mathrm{s}^{-1}$. This is consistent with the upper limit obtained from our spectral analysis $\left(L_{\text {th,bol }} \lesssim 2 \times\right.$ $10^{33} \mathrm{erg} \mathrm{s}^{-1}$; Section 2.2). Any soft thermal emission from Swift J1749.4-2807 suffers considerably from the high absorption column density. Our observational limits therefore do not place strong constraints on the thermal state of the neutron star.

We note that the low estimated long-term averaged massaccretion rate may not reconcile with the expected mass-transfer rate from the companion found in standard binary evolution calculations (e.g., Podsiadlowski et al. 2002; King \& Wijnands 2006). For a Roche-lobe filling main-sequence star of $M_{\mathrm{d}} \simeq$ $0.6 M_{\odot}$ and an orbital period of $P_{\text {orb }}=8.8 \mathrm{hr}$, the expected 
Table 2

Quiescent Spectral Properties of Accreting Millisecond X-Ray Pulsars

\begin{tabular}{|c|c|c|c|c|c|}
\hline Source & $\begin{array}{c}D \\
(\mathrm{kpc})\end{array}$ & $\begin{array}{c}L_{\mathrm{X}} \\
\left(\mathrm{erg} \mathrm{s}^{-1}\right)\end{array}$ & $\begin{array}{c}k T \\
(\mathrm{keV})\end{array}$ & Thermal Fraction & Reference \\
\hline \multicolumn{6}{|c|}{ Detections } \\
\hline $\mathrm{Aq} 1 \mathrm{X}-1^{\dagger}$ & 5 & $\simeq 1 \times 10^{33}$ & $\simeq 0.12$ & $\gtrsim 50 \%$ & $1-4$ \\
\hline SAX J1748.9-2021 ${ }^{\dagger}$ & 8.5 & $\simeq 1 \times 10^{33}$ & $\simeq 0.09$ & $\geq 60 \%$ & 5,6 \\
\hline Swift J1749.4-2807 & 6.7 & $\simeq 7 \times 10^{32}$ & $<0.10$ & $<55 \%$ & 7 \\
\hline IGR J17498-2921 & 7.6 & $\simeq 2 \times 10^{32}$ & $\lesssim 0.08$ & $\ldots$ & 8 \\
\hline IGR J00291+5934 & 4 & $\simeq 1 \times 10^{32}$ & $\simeq 0.07$ & $\simeq 40 \%$ & $9-12$ \\
\hline SAX J1808.4-3658 & 2.5 & $\simeq 8 \times 10^{31}$ & $<0.03$ & $<5 \%$ & $12-17$ \\
\hline XTE J0929-314 & 10 & $\simeq 7 \times 10^{31}$ & $<0.05$ & $<30 \%$ & 18,19 \\
\hline \multicolumn{6}{|c|}{ Non-detections } \\
\hline IGR J17511-3057 & 6.9 & $\lesssim 4 \times 10^{33}$ & $<0.10$ & $\ldots$ & 20 \\
\hline Swift J1756.9-258 & 8 & $\widetilde{\Sigma}_{2} \times 10^{33}$ & $<0.08$ & $\ldots$ & 20,21 \\
\hline HETE J1900.1-2455 ${ }^{\dagger}$ & 3.6 & $\lesssim 5 \times 10^{32}$ & $<0.06$ & $\cdots$ & 20,22 \\
\hline XTE J1814-338 & 8 & $\widetilde{\Sigma}_{2} \times 10^{32}$ & $<0.07$ & $\ldots$ & 12 \\
\hline XTE J1751-305 & 8 & $\stackrel{\lesssim}{\lesssim} \times 10^{32}$ & $<0.07$ & $\ldots$ & 19 \\
\hline XTE J1807-294 & 8 & $\widetilde{\Sigma}^{\lesssim} 4 \times 10^{31}$ & $<0.05$ & $\ldots$ & 18 \\
\hline NGC $6440 \mathrm{X}-2$ & 8.5 & $\widetilde{\Sigma}_{2} \times 10^{31}$ & $<0.03$ & $\ldots$ & 20,23 \\
\hline
\end{tabular}

Notes. The sources are arranged according to descending quiescent X-ray luminosity. The three that are marked by a dagger are "intermittent" and do not persistently show X-ray pulsations during outburst. The quoted temperatures are for an observer at infinity and assume a pure-H neutron star atmosphere model for the thermal emission. $L_{\mathrm{X}}$ denotes the total average $0.5-10 \mathrm{keV}$ quiescent luminosity (i.e., including thermal and non-thermal contributions) assuming the listed distances $(D)$. The fifth column gives the fractional contribution of the thermal component to the total unabsorbed $0.5-10 \mathrm{keV}$ model flux. Due to limited statistics, the spectral shape of IGR J17498-2921 cannot be constrained with the current available data.

References. (1) Rutledge et al. 2001; (2) Rutledge et al. 2002; (3) Campana \& Stella 2003; (4) Cackett et al. 2011; (5) in't Zand et al. 2001; (6) Cackett et al. 2005; (7) This work, (8) Jonker et al. 2011; (9) Jonker et al. 2005; (10) Jonker et al. 2008; (11) Campana et al. 2008; (12) Heinke et al. 2009; (13) Stella et al. 2000; (14) Dotani et al. 2000; (15) Wijnands et al. 2002; (16) Campana et al. 2002; (17) Heinke et al. 2007; (18) Campana et al. 2005; (19) Wijnands et al. 2005b; (20) Haskell et al. 2012; (21) Patruno et al. 2010; (22) Degenaar et al. 2007; (23) Heinke et al. 2010.

mass-transfer rate averaged over the lifetime of the binary $\left(\simeq 10^{9}\right.$ yr) would be on the order of $\dot{M}_{\mathrm{d}}=\langle\dot{M}\rangle \simeq 2 \times$ $10^{-11} M_{\odot} \mathrm{yr}^{-1}$ (see Verbunt 1993, for a review). This is a factor $\simeq 25$ higher than the mass-accretion rate that we estimated based on X-ray observations of Swift J1749.4-2807. However, the mass-transfer rate from the donor star may not be equal to the mass-accretion rate onto the neutron star, e.g., because the pulsar's propeller expels matter and prevents it from accreting onto its surface (Illarionov \& Sunyaev 1975). Furthermore, the $\mathrm{X}$-ray emission may not be a good tracer of the mass-transfer rate, and the outburst behavior observed in the past decade may not be representative for the long-term accretion history of the binary. Therefore, both estimates are subject to uncertainties.

\subsection{The Origin of the Quiescent X-Ray Emission}

In addition to a soft thermal component, many neutron star LMXBs display hard emission tails that dominate the quiescent $\mathrm{X}$-ray spectrum at energies above $\simeq 2-3 \mathrm{keV}$. These can typically be fit with a simple power law of index $\Gamma \simeq 1-2$, which is similar to our findings for Swift J1749.4-2807 ( $\Gamma \simeq 1.6$ ). This (non-thermal) component is not accounted for by deep crustal heating. Proposed mechanisms include residual accretion onto the magnetic field of the neutron star, while non-accretion scenarios explain the power-law emission as a shock from the pulsar wind colliding with matter flowing out of the donor star, or the pulsar wind mechanism itself (e.g., Campana et al. 1998; Rutledge et al. 2001).

It is interesting to compare the quiescent properties of Swift J1749.4-2807 to that of the other AMXPs. For this purpose, we have summarized the basic quiescent spectral properties of all
14 currently known sources in Table 2 (in order of decreasing luminosity). To date, seven have been detected in quiescence and spectral analysis could be performed for six of these. Aql X-1 and SAX J1748.9-2021 are both intermittent AMXPs, which show pulsations only sporadically during their outbursts (Altamirano et al. 2008; Casella et al. 2008). These two have the highest quiescent luminosities of $\simeq 1 \times 10^{33} \mathrm{erg} \mathrm{s}^{-1}$. Although both show spectral variability in quiescence, typically $>50 \%$ of the emission can be ascribed to a thermal component (Rutledge et al. 2002; Campana \& Stella 2003; Cackett et al. 2005, 2011).

The other AMXPs are fainter $\left(\simeq 1 \times 10^{32} \mathrm{erg} \mathrm{s}^{-1}\right.$ and below $)$ and those with well-studied spectra (IGR J00291+5934, SAX J1808.4-3658, and XTE J0929-314) display power-lawdominated emission with $\lesssim 40 \%$ attributable to a thermal component (Table 2). These sources also show evidence for intensity variations by a factor of a few, within single observations or between different epochs (Wijnands et al. 2005b; Jonker et al. 2008; Heinke et al. 2009).

Swift J1749.4-2807 is relatively bright compared to most AMXPs, but its spectral properties are not notably different: It shows a strong power-law component that can account for at least $45 \%$ of the quiescent emission. Swift J1749.4-2807 is similarly bright as Aql X-1 and SAX J1748.9-2021. These three AMXPs have the largest orbital periods ( $\gtrsim 9 \mathrm{hr}$ ), whereas the others have shorter orbital periods of ( $\lesssim 4 \mathrm{hr})$. Unlike the other AMXPs that have been detected in quiescence on multiple occasions, Swift J1749.4-2807 does not show evidence for (strong) variations in its quiescent intensity between different epochs. However, the source was located far off-axis in the archival observations of 2000 and 2006, prohibiting an accurate determination of its quiescent flux (Wijnands et al. 2009). 
It has been noted that the quiescent spectra of the AMXPs are relatively hard compared to those of non-pulsating neutron star LMXBs (Campana et al. 2005, 2008; Wijnands et al. 2005b; Heinke et al. 2009). Jonker et al. (2004) studied a sample of 15 neutron stars and found a possible correlation between the fractional contribution of the non-thermal component to the total $0.5-10 \mathrm{keV}$ quiescent flux. In the luminosity regime of $\simeq 10^{33} \mathrm{erg} \mathrm{s}^{-1}$, the power-law contribution appears to be at its minimum and typically $<30 \%$. This is lower than the $>45 \%$ non-thermal emission that we infer from our XMM-Newton data. Although the high-absorption column density strongly obscures any soft emission from Swift J1749.4-2807 and a thermal component may still contribute up to $\simeq 55 \%$, its spectrum therefore indeed appears to be relatively hard compared to non-pulsating LMXBs that have similar quiescent $0.5-10 \mathrm{keV}$ luminosities.

Several other LMXBs that were observed after the study of Jonker et al. (2004), and are similarly bright as Swift J1749.4-2807, indeed also show predominantly thermal X-ray spectra with a power-law tail that contributes $\lesssim 20 \%$ to the total quiescent emission (e.g., Degenaar et al. 2009; Servillat et al. 2012; Lowell et al. 2012). So far, the only outlier is the non-pulsating LMXB EXO 1745-248 in the globular cluster Terzan 5. This neutron star also has a quiescent luminosity of $\simeq 10^{33} \mathrm{erg} \mathrm{s}^{-1}$, but displays a similarly hard quiescent spectrum as the AMXPs, with $\gtrsim 40 \%$ attributable to a non-thermal component (Wijnands et al. 2005a; Degenaar \& Wijnands 2012).

The fact that several of the AMXPs display particularly prominent power-law emission in quiescence suggests a possible connection with the magnetic field of the neutron star. However, the $11 \mathrm{~Hz}$ X-ray pulsar in Terzan 5 is thought to have a relatively strong magnetic field ( $B \simeq 10^{9-10} \mathrm{G}$ ), but its quiescent emission is fully thermal and does not require the addition of a power-law component (Degenaar \& Wijnands 2011a, 2011b). Recent observations provide strong evidence that at least in some (non-pulsating) quiescent LMXBs, the non-thermal power-law emission is related to a continued accretion flow that reaches the neutron star surface (Cackett et al. 2010; Fridriksson et al. 2011). The effect of residual accretion could be a viable explanation for the relatively luminous and hard quiescent spectrum of Swift J1749.4-2807.

In addition to causing quiescent variability, residual accretion may leave detectable signatures in the X-ray emission. If the observed quiescent X-ray luminosity of Swift J1749.4-2807 can be ascribed to an accretion flow that reaches the surface of the neutron star, then the implied quiescent mass accretion rate of $\left\langle\dot{M}_{\mathrm{q}}\right\rangle \simeq 10^{-13} M_{\odot} \mathrm{yr}^{-1}$ may allow metals to be maintained in the atmosphere (Bildsten et al. 1992; Brown et al. 1998). This could cause the emergent spectrum to deviate from a pure $\mathrm{H}$-atmosphere and give rise to gravitationally redshifted metal absorption lines. Comparing the spectral data with neutron star atmospheres of different composition has the potential to test this (Ho \& Heinke 2009; Servillat et al. 2012).

N.D. is supported by NASA through the Hubble Postdoctoral Fellowship grant number HST-HF-51287.01-A from the Space Telescope Science Institute, which is operated by the Association of Universities for Research in Astronomy, Incorporated, under the NASA contract NAS5-26555. A.P. acknowledges support from the Netherlands Organization for Scientific research (NWO) through the Veni-fellowship program, and R.W. is supported by a European Research Council (ERC) starting grant. N.D. thanks Jon M. Miller for stimulating discussions. The authors are grateful to the anonymous referee for thoughtful comments that helped improve this manuscript.

Facility: XMM (EPIC)

\section{REFERENCES}

Altamirano, D., Casella, P., Patruno, A., Wijnands, R., \& van der Klis, M. 2008, ApJ, 674, L45

Altamirano, D., Cavecchi, Y., Patruno, A., et al. 2011, ApJ, 727, L18

Altamirano, D., Wijnands, R., van der Klis, M., et al. 2010, ATel, 2565

Anders, E., \& Grevesse, N. 1989, Geochim. Cosmochim. Acta, 53, 197

Arnaud, K. A. 1996, in ASP Conf. Ser. 101, Astronomical Data Analysis Software and Systems V, ed. G. H. Jacoby \& J. Barnes (San Francisco, CA: ASP), 17

Bassa, C. G., Jonker, P. G., Steeghs, D., \& Torres, M. A. P. 2009, MNRAS, 399,2055

Beardmore, A. P., Godet, O., \& Sakamoto, T. 2006, GRB Coordinates Network, 5209

Bildsten, L., Salpeter, E. E., \& Wasserman, I. 1992, ApJ, 384, 143

Brown, E. F., Bildsten, L., \& Rutledge, R. E. 1998, ApJ, 504, L95

Cackett, E. M., Brown, E. F., Miller, J. M., \& Wijnands, R. 2010, ApJ, 720,1325

Cackett, E. M., Fridriksson, J. K., Homan, J., Miller, J. M., \& Wijnands, R. 2011, MNRAS, 414, 3006

Cackett, E. M., Wijnands, R., Heinke, C. O., et al. 2005, ApJ, 620, 922

Campana, S., Colpi, M., Mereghetti, S., Stella, L., \& Tavani, M. 1998, Astron. Astrophys. Rev., 8, 279

Campana, S., Ferrari, N., Stella, L., \& Israel, G. L. 2005, A\&A, 434, L9

Campana, S., \& Stella, L. 2003, ApJ, 597, 474

Campana, S., Stella, L., Gastaldello, F., et al. 2002, ApJ, 575, L15

Campana, S., Stella, L., Israel, G., \& D’Avanzo, P. 2008, ApJ, 689, L129

Casella, P., Altamirano, D., Patruno, A., Wijnands, R., \& van der Klis, M. 2008, ApJ, 674, L41

Chen, W., Shrader, C. R., \& Livio, M. 1997, ApJ, 491, 312

Church, M. J., Dotani, T., Balucinska-Church, M., et al. 1997, ApJ, 491, 388

D’Avanzo, P., Campana, S., Muñoz-Darias, T., et al. 2011, A\&A, 534, A92

Degenaar, N., Brown, E. F., \& Wijnands, R. 2011, MNRAS, 418, L152

Degenaar, N., \& Wijnands, R. 2011a, MNRAS, 414, L50

Degenaar, N., \& Wijnands, R. 2011b, MNRAS, 412, L68

Degenaar, N., \& Wijnands, R. 2012, MNRAS, 422, 581

Degenaar, N., Wijnands, R., Campana, S., et al. 2007, ATel, 1098

Degenaar, N., Wijnands, R., Wolff, M. T., et al. 2009, MNRAS, 396, L26

Dotani, T., Asai, K., \& Wijnands, R. 2000, ApJ, 543, L145

Ferrigno, C., Bozzo, E., Falanga, M., et al. 2011, A\&A, 525, A48

Fridriksson, J. K., Homan, J., Wijnands, R., et al. 2011, ApJ, 736, 162

Gupta, S., Brown, E. F., Schatz, H., Möller, P., \& Kratz, K.-L. 2007, ApJ, 662,1188

Haensel, P., \& Zdunik, J. L. 2008, A\&A, 480, 459

Halpern, J. 2006, GRB Coordinates Network, 5210

Haskell, B., Degenaar, N., \& Ho, W. C. G. 2012, MNRAS, 424, 93

Heinke, C. O., Altamirano, D., Cohn, H. N., et al. 2010, ApJ, 714, 894

Heinke, C. O., Jonker, P. G., Wijnands, R., Deloye, C. J., \& Taam, R. E. 2009, ApJ, 691, 1035

Heinke, C. O., Jonker, P. G., Wijnands, R., \& Taam, R. E. 2007, ApJ, 660, 1424

Heinke, C. O., Rybicki, G. B., Narayan, R., \& Grindlay, J. E. 2006, ApJ, 644,1090

Ho, W. C. G., \& Heinke, C. O. 2009, Nature, 462, 71

Illarionov, A. F., \& Sunyaev, R. A. 1975, A\&A, 39, 185

in't Zand, J. J. M., van Kerkwijk, M. H., Pooley, D., et al. 2001, ApJ, 563, L41 Jonker, P. G., Campana, S., Steeghs, D., et al. 2005, MNRAS, 361, 511

Jonker, P. G., Galloway, D. K., McClintock, J. E., et al. 2004, MNRAS, 354,666

Jonker, P. G., Torres, M. A. P., \& Steeghs, D. 2008, ApJ, 680, 615

Jonker, P. G., Torres, M. A. P., Steeghs, D., \& Chakrabarty, D. 2011, ATel, 3559

King, A. R., \& Wijnands, R. 2006, MNRAS, 366, L31

Lattimer, J. M., \& Prakash, M. 2001, ApJ, 550, 426

Lowell, A. W., Tomsick, J. A., Heinke, C. O., et al. 2012, ApJ, 749, 111

Markwardt, C. B., \& Strohmayer, T. E. 2010, ApJ, 717, L149

Markwardt, C. B., Strohmayer, T. E., Swank, J. H., Pereira, D., \& Smith, E. 2010, ATel, 2576

Page, D., Lattimer, J. M., Prakash, M., \& Steiner, A. W. 2004, ApJS, 155, 623

Patruno, A. 2010, ApJ, 722, 909

Patruno, A., Altamirano, D., \& Messenger, C. 2010, MNRAS, 403, 1426

Pavan, L., Chenevez, J., Bozzo, E., et al. 2010, ATel, 2548

Podsiadlowski, P., Rappaport, S., \& Pfahl, E. D. 2002, ApJ, 565, 1107 
Rutledge, R. E., Bildsten, L., Brown, E. F., Pavlov, G. G., \& Zavlin, V. E. 1999, ApJ, 514, 945

Rutledge, R. E., Bildsten, L., Brown, E. F., Pavlov, G. G., \& Zavlin, V. E. 2001, ApJ, 559, 1054

Rutledge, R. E., Bildsten, L., Brown, E. F., Pavlov, G. G., \& Zavlin, V. E. 2002, ApJ, 577, 346

Servillat, M., Heinke, C. O., Ho, W. C. G., et al. 2012, MNRAS, 423, 1556

Stella, L., Campana, S., Mereghetti, S., Ricci, D., \& Israel, G. L. 2000, ApJ, 537, L115

Strüder, L., Briel, U., Dennerl, K., et al. 2001, A\&A, 365, L18

Tokunaga, A. T. 2000, in Infrared Astronomy, ed. A. N. Cox (New York: Springer), 143

Turner, M. J. L., Abbey, A., Arnaud, M., et al. 2001, A\&A, 365, L27

Verbunt, F. 1993, ARA\&A, 31, 93
Watson, M. G., Schröder, A. C., Fyfe, D., et al. 2009, A\&A, 493, 339

Wijnands, R. 2006, in Trends in Pulsar Research, ed. J. A. Lowry (New York: Nova Science Publishers), 53

Wijnands, R., Heinke, C. O., Pooley, D., et al. 2005a, ApJ, 618, 883

Wijnands, R., Homan, J., Heinke, C. O., Miller, J. M., \& Lewin, W. H. G. 2005b, ApJ, 619, 492

Wijnands, R., Kuiper, L., in 't Zand, J., et al. 2002, ApJ, 571, 429

Wijnands, R., Miller, J. M., Markwardt, C., Lewin, W. H. G., \& van der Klis, M. 2001, ApJ, 560, L159

Wijnands, R., Nowak, M., Miller, J. M., et al. 2003, ApJ, 594, 952

Wijnands, R., Rol, E., Cackett, E., Starling, R. L. C., \& Remillard, R. A. 2009, MNRAS, 393, 126

Yakovlev, D. G., Levenfish, K. P., \& Haensel, P. 2003, A\&A, 407, 265

Yang, Y. J., Russell, D. M., Wijnands, R., et al. 2010, ATel, 2579 